\title{
EXTENDED X-RAY EMISSION FROM PLANETARY NEBULAE
}

\author{
H.C. KREYSING, C. DIESCH, J. ZWEIGLE, R. STAUBERT and M. GREWING \\ Astronomisches Institut der Universität Tübingen, Waldhäuserstr. 64, D-7400 Tübingen, \\ Germany
}

We present first results from the ROSAT All Sky Survey on X-ray emission of planetary nebulae $(\mathrm{PNe})$. For the first time extended X-ray emission from $\mathrm{PNe}$ was detected. This is the case for NGC 6543, NGC 6853, A 12, NGC 4361 (and LoTr $5)$. X-ray emission compatible with a point source was detected from $\mathrm{BD}+30^{\circ} 3639$, however, the spectral distribution of the X-ray photons is leading to temperatures beyond $210^{6} \mathrm{~K}$. Thus in all cases, with the possible exception of LoTr 5 , the central star of the PNe can be excluded as the main source of the observed X-ray emission. X-ray images and ROSAT spectra for all detected PNe are presented. The best observed PN in X-ray emission is NGC 6543. Due to the close vicinity to the north ecliptic pole, this object was regularly observed, every 90 minutes during the whole half year of the ROSAT All Sky Survey, resulting in $41 \mathrm{ksec}$ of integration time. In addition NGC 6543 was observed in a $50 \mathrm{ksec}$ pointed observation to the north ecliptic pole, taken in June 1990 during the calibration phase (Kreysing et al. 1992). A comparison of the semi-ring-like distribution of the X-ray emission of NGC 6543 with optical CCD-images shows, that most of the X-ray emission seems to originate from the boundary region between the nebula and the halo. Neither the central star nor the hot wind from the central star wind is the main source of the X-ray emission, as proposed by the interacting stellar wind model (Kwok 1982). An alternative model employing a possible coronal heating mechanism has been discussed by Kreysing (1992); accoustic waves, travelling outward from the nebula, encounter a sudden density decline at the boundary to the halo. As a consequence the waves degenerate into shock waves, dissipating their energy in a thin region of only some $1015 \mathrm{~cm}$ into the ambient medium.

\begin{tabular}{llllll}
\hline & $\mathrm{d}[\mathrm{kpc}]$ & {$[\mathrm{cts} / \mathrm{sec}]$} & $\mathrm{T}_{x}[\mathrm{~K}]$ & $\mathrm{N}_{H}\left[\mathrm{~cm}^{-3}\right]$ & $\mathrm{L}_{x}[\mathrm{erg} / \mathrm{sec}]^{*}$ \\
\hline \hline BD+30 3639 & $0.2-2.8$ & $0.18 \pm 0.03$ & $2.510^{6}$ & $1.410^{21}$ & $4.310^{32}-8.510^{34}$ \\
NGC 6543 & 1.2 & $(8.1 \pm 0.4) 10^{-3}$ & $1.710^{6}$ & $6.910^{20}$ & $(1.7 \pm 0.1) 10^{32}$ \\
A 12 & 2.4 & $0.10 \pm 0.02$ & $3.310^{6}$ & - & $6.910^{32}-2.310^{34}$ \\
NGC 4361 & 0.86 & $0.05 \pm 0.02$ & $<2.010^{5}$ & $4.910^{20}$ & $(2.0 \pm 0.8) 10^{34}$ \\
LoTr 5 & 0.4 & $0.04 \pm 0.01$ & $<2.010^{5}$ & $<10^{18}$ & $(1.3 \pm 0.3) 10^{31}$ \\
\hline
\end{tabular}

* the X-ray luminosity is given in range $0.1-2.4 \mathrm{keV}$

\section{References}

Kreysing H.C. et al. 1992, As.\& Ap., accepted.

Kreysing H.C, 1992, submitted, Ph.D. thesis, University of Tübingen

Kwok S. 1982, ApJ. 258, 280 